\title{
Morphological response contributes to patient selection for rescue liver resection in chemotherapy patients with initially un-resectable colorectal liver metastasis
}

\author{
KOICHI SUZUKI ${ }^{1}$, YUTA MUTO ${ }^{1}$, KOSUKE ICHIDA $^{1}$, TARO FUKUI ${ }^{1}$, YUJI TAKAYAMA ${ }^{1}$, NAO KAKIZAWA ${ }^{1}$, \\ TAKAHARU KATO $^{1}$, FUMI HASEGAWA ${ }^{1}$, FUMIAKI WATANABE ${ }^{1}$, YUJI KANEDA ${ }^{1}$, RINA KIKUKAWA ${ }^{1}$, \\ MASAAKI SAITO ${ }^{1}$, SHINGO TSUJINAKA ${ }^{1}$, KAZUSHIGE FUTSUHARA ${ }^{1}$, OSAMU TAKATA ${ }^{1}$, HIROSHI NODA ${ }^{1}$, \\ YASUYUKI MIYAKURA ${ }^{1}$, HIROKAZU KIYOZAKI ${ }^{1}$, FUMIO KONISHI $^{2}$ and TOSHIKI RIKIYAMA ${ }^{1}$ \\ ${ }^{1}$ Department of Surgery, Saitama Medical Center, Jichi Medical University, Saitama 330-8503; \\ ${ }^{2}$ Department of Surgery, Nerima-Hikarigaoka Hospital, Tokyo 179-0072, Japan
}

Received March 7, 2016; Accepted February 13, 2017

DOI: $10.3892 / 01.2017 .6338$

\begin{abstract}
Morphological response is considered an improved surrogate to the Response Evaluation Criteria in Solid Tumors (RECIST) model with regard to predicting the prognosis for patients with colorectal liver metastases. However, its use as a decision-making tool for surgical intervention has not been examined. The present study assessed the morphological response in 50 patients who underwent chemotherapy with or without bevacizumab for initially un-resectable colorectal liver metastases. Changes in tumor morphology between heterogeneous with uncertain borders and homogeneous with clear borders were defined as an optimal response (OR). Patients were also assessed as having an incomplete response (IR), and an absence of marked changes was assessed as no response (NR). No significant difference was observed in progression-free survival (PFS) between complete response/partial response (CR/PR) and stable disease/progressive disease (SD/PD), according to RECIST. By contrast, PFS for OR/IR patients was significantly improved compared with that for NR patients (13.2 vs. 8.7 months; $\mathrm{P}=0.0426$ ). Exclusion of PD enhanced the difference in PFS between OR/IR and NR patients (15.1 vs. 9.3 months; $\mathrm{P}<0.0001$ ), whereas no difference was observed between CR/PR and SD. The rate of OR and IR in patients treated with bevacizumab was $47.4 \%$ (9/19), but only $19.4 \%$ (6/31) for patients that were not administered bevacizumab. Comparison of the survival curves between OR/IR and NR patients revealed similar
\end{abstract}

Correspondence to: Dr Koichi Suzuki, Department of Surgery, Saitama Medical Center, Jichi Medical University, 1-847 Amanuma, Omiya, Saitama 330-8503, Japan

E-mail: ksuzbnhm@yahoo.co.jp

Key words: rescue liver resection, morphological response, bevacizumab, colorectal liver metastasis, Response Evaluation Criteria in Solid Tumors survival rates at 6 months after chemotherapy, but the groups exhibited different survival rates subsequent to this period of time. Patients showing OR/IR within 6 months appeared to be oncologically stable and could be considered as candidates for surgical intervention, including rescue liver resection. Comparing the pathological and morphological features of the tumor with representative optimal response, living tumor cells were revealed to be distributed within the area of vascular reconstruction induced by bevacizumab, resulting in a predictive value for prognosis in the patients treated with bevacizumab. The present findings provided the evidence for physicians to consider patients with previously un-resectable metastatic colorectal cancer as candidates for surgical treatment. Morphological response is a useful decision-making tool for evaluating these patients for rescue liver resection following chemotherapy.

\section{Introduction}

Recent improvements in chemotherapy, including the use of biological agents, have been shown to prolong the survival of patients with metastatic colorectal cancer (mCRC), with a recorded median overall survival time of 30 months $(1,2)$. For patients with $\mathrm{mCRC}$ and liver metastasis, liver resection combined with systemic therapies resulted in a 5-year survival rate of $25-40 \%$ (3). Combination regimens using various biological agents with cytotoxic chemotherapy achieved high response rates and a reduced tumor size (4). This enables liver resection for $\mathrm{mCRC}$ patients with borderline resectable, as well as un-resectable, liver metastasis.

Tumor response to chemotherapy is the initial step in the selection of patients who are most likely to benefit from surgery. Changes in the characteristics of liver metastases may be assessed with respect to surgical and oncological viewpoints. In addition to the technical considerations, the ability of the tumor to be resected and its biology are the most important factors that determine the outcome of the patients. Candidates for tumor resection of liver metastases should have surgically resectable masses and be oncologically stable. 
The patients should also be unlikely to relapse within a short period of time following surgery (5-11).

The Response Evaluation Criteria in Solid Tumors (RECIST) model is widely used to evaluate tumor response, however, since its introduction, there has been increasing concern regarding the use of traditional tumor response criteria $(12,13)$. This is due to RECIST being limited in its application in assessing the response of tumors to biological agents that exhibit a cytostatic mechanism of action. For patients with metastatic gastrointestinal stromal tumors, RECIST using anatomical information only (such as tumor size), has been shown to significantly underestimate the initial tumor response to imatinib (14). This is since patients that exhibit a stable response to imatinib have a similar outcome to those who achieve a complete response (CR) or partial response (PR) when evaluated using RECIST (15). Several studies have shown that morphological response is an improved alternative to RECIST for predicting the outcome of patients with colorectal liver metastases (16-18). However, the potential clinical application of morphological evaluation has not been attempted for the selection of patients most likely to benefit from surgery. The present study examined whether evaluations that included morphological criteria were useful in selecting the best therapeutic strategy for patient treatment.

\section{Patients and methods}

Patients. A total of 50 patients with $\mathrm{mCRC}$ and unresectable liver metastasis were recruited for the present retrospective study. The patients had histologically confirmed and measurable mCRC. Each patient underwent oxaliplatin-based chemotherapy, with or without bevacizumab, between May 2008 and November 2012 at the Saitama Medical Center (Jichi Medical University, Saitama, Japan). The present study was approved by the Research Ethics Committee of Jichi Medical University.

Imaging analysis. Tumor morphology was assessed using enhanced computed tomography (CT) and characterized according to the criteria previously described (16): Group 1, homogeneous low attenuation with a thin, sharply-defined tumor-liver interface; group 3 , heterogeneous attenuation with a thick, poorly-defined tumor-liver interface; and group 2 , intermediate morphology that could be rated as either group 1 or 3 . A change in morphology between group 3 or 2 to group 1 was defined as an optimal response (OR; Fig. 1), and a group 3 to group 2 change was defined as an incomplete response (IR). The absence of marked changes in tumor morphology was defined as no response (NR; Fig. 1). In patients with multiple tumors, morphological response was assigned based on changes observed in the majority of the tumors. Response to chemotherapy was also determined using RECIST.

Statistical analysis. Fisher's exact test was used to examine the association between two categorical variables. Continuous comparison of the variables between two groups was performed. Unpaired t-tests were used for those variables that followed a normal distribution, and the non-parametric Mann-Whitney-Wilcoxon test was used for those variables that did not follow a normal distribution. $\mathrm{P}<0.05$ was considered to indicate a statistically significant difference. Values are shown as the mean \pm standard error. Progression-free survival (PFS) and overall survival (OS) data were plotted as Kaplan-Meier curves, and the differences among the groups were compared using a log-rank test.

\section{Results}

Characteristics of patients. The present study included 34 men and 16 women with a median age of 65 years (range, 45-83 years). A total of 28 patients presented with primary colon tumors, while the other 22 patients were diagnosed with tumors of the rectum. Liver metastasis was detected simultaneously in 34 patients and metachronously in 16 patients. Solitary liver metastases were observed in 19 patients and multiple metastases were observed in 31 patients. A total of 30 patients presented with metastasis in extrahepatic regions, including 10 in the lymph nodes, 9 in the lung, 5 in the intra-peritoneum, 2 in the bone, 2 in the pelvic node at the anastomotic site, one in the spleen and one in the ovarian. The size of the largest metastasis ranged from 9 to $140 \mathrm{~mm}$, with a median size of $47 \mathrm{~mm}$. All patients underwent oxaliplatin-based chemotherapy with or without bevacizumab as the first line of treatment. The treatment regimens were mFOLFOX6 in 15 patients, which included $200 \mathrm{mg} / \mathrm{m}^{2}$ folinic acid, $400 \mathrm{mg} / \mathrm{m}^{2}$ 5-FU and $85 \mathrm{mg} / \mathrm{m}^{2}$ oxaliplatin on day 1 , followed by $46 \mathrm{~h}$ of continuous infusion with $2,400 \mathrm{mg} / \mathrm{m}^{2} 5$-FU on days 1 and 2 and XELOX in the remaining 35 patients, which consisted of $2,000 \mathrm{mg} / \mathrm{m}^{2}$ capecitabine on days $1-15$ ) and $130 \mathrm{mg} / \mathrm{m}^{2}$ oxaliplatin on day 1 . A total of 19 patients were also treated with bevacizumab, anti-vascular endothelial growth factor (VEGF) antibody: For one patient treated with mFOLFOX6 regimen, $5 \mathrm{mg} / \mathrm{kg}$ bevacizumab was administrated on day 1 and q14d, and for 18 patients treated with XELOX regimen, $7.5 \mathrm{mg} / \mathrm{kg}$ bevacizumab was administrated on day 1 and q21d.

Morphological response rate. The morphological response rate following treatment with or without bevacizumab is shown in Table I. There were 14 responders (28.0\%), including 7 patients with an OR (14.0\%) and 7 patients with an IR $(14.0 \%)$. A total of 36 patients $(72.0 \%)$ showed NR. The rate of patients classified as OR/IR who were treated with bevacizumab was $47.4 \%(9 / 19)$, whereas for patients who did not receive bevacizumab, the rate was $19.4 \%(6 / 31)$.

CT evaluations according to RECIST and morphological criteria. Using RECIST, 10 patients had a CR/PR, 27 had stable disease (SD) and 13 had progressive disease (PD). No significant difference was observed in PFS time between those patients who were classified as CR/PR and those patients who had SD/PD (10.9 months for CR/PR vs. 8.6 months for $\mathrm{SD} / \mathrm{PD}$; $\mathrm{P}=0.2604$; Fig. $2 \mathrm{~A}$ ). According to the morphological criteria, 14 patients were classified as having OR/IR, while 36 patients had NR. PFS time for patients showing OR/IR was significantly improved when compared with patients showing NR (13.2 months for OR/IR vs. 8.7 months for NR; $\mathrm{P}=0.0426$; Fig. 2B). For those patients who showed PD, they also had a short PFS period (4.0 months; $n=16$ ) compared with the SD and $C R / P R$ groups $(\mathrm{P}<0.0001)$. Therefore, these patients were classified as group 1 and used as a comparison to patients in 
Table I. Morphological response rate according to treatment with or without bevacizumab.

\begin{tabular}{lcccc}
\hline Treatment & Patients, $\mathrm{n}$ & Optimal response, $\mathrm{n}(\%)$ & Incomplete response, $\mathrm{n}(\%)$ & No response, $\mathrm{n}(\%)$ \\
\hline $\begin{array}{l}\text { Total } \\
\text { Chemotherapy }\end{array}$ & 50 & $7 / 50(14.0)$ & $7 / 50(14.0)$ & $36 / 50(72.0)$ \\
$\begin{array}{l}\text { with bevacizumab } \\
\begin{array}{l}\text { Chemotherapy } \\
\text { without bevacizumab }\end{array}\end{array}$ & 19 & $5 / 19(26.3)$ & $4 / 19(21.1)$ & $10 / 19(52.6)$ \\
\end{tabular}

Optimal response

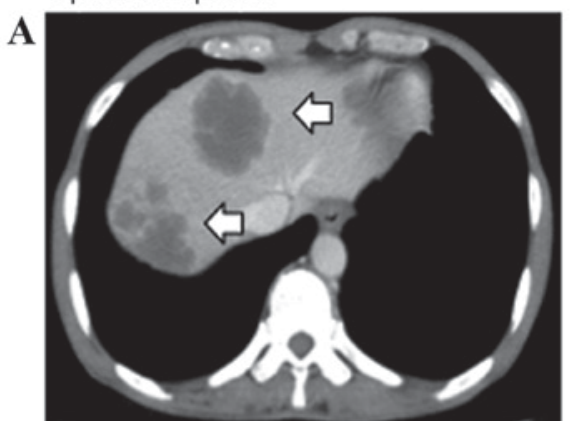

C

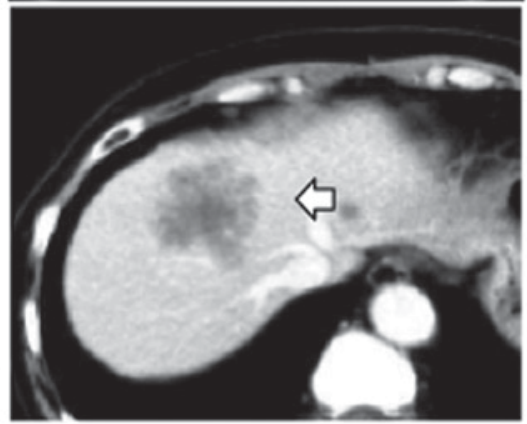

No response

E

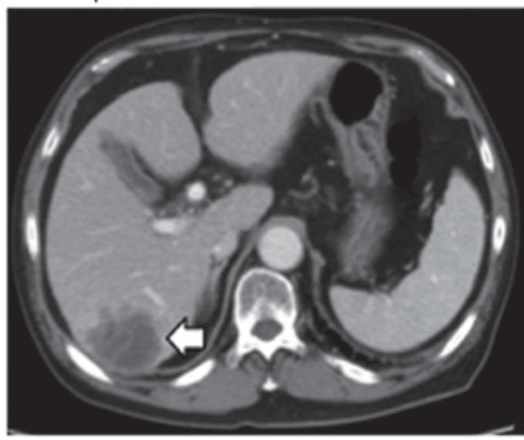

G

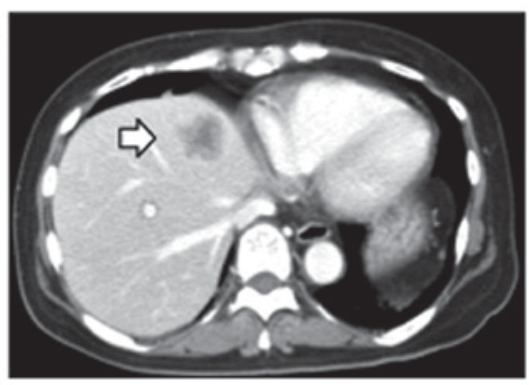

B

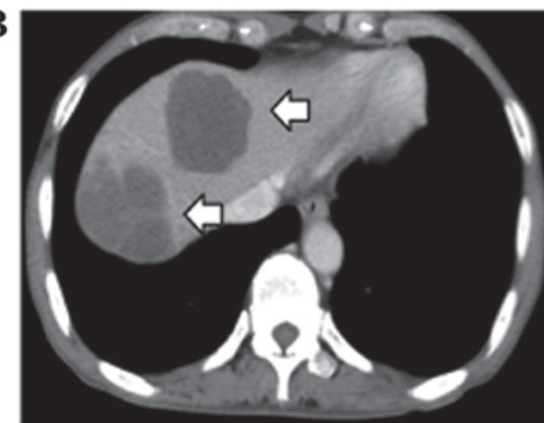

D

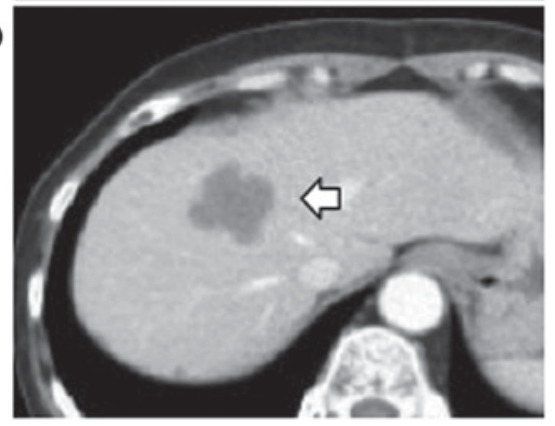

F

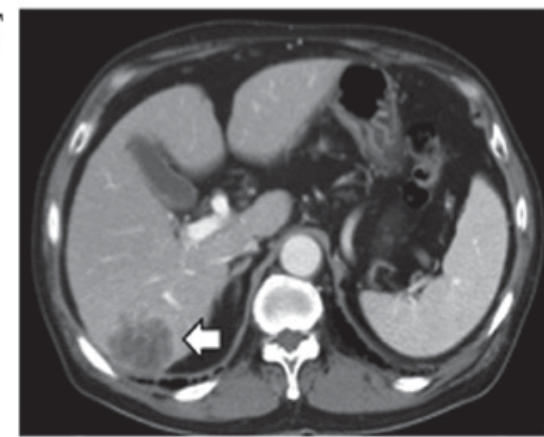

H

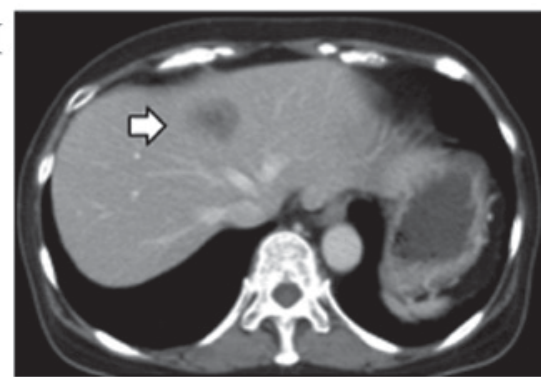

Figure 1. Change in morphology according to morphological response. Optimal response was defined as the change in morphology from the tumor harboring heterogeneous attenuation with a thick, poorly-defined tumor-liver interface (group 3) to homogeneous low attenuation with a thin, sharply-defined tumor-liver interface (group 1). No response was defined as absence of marked changes in morphology (no change from group 3). Arrows highlight metastatic liver tumors in which morphology were estimated prior and subsequent to chemotherapy. (A) Representative image of case 1 with optimal response prior to chemotherapy and (B) subsequent to chemotherapy. (C) Representative image of case 2 with optimal response prior to chemotherapy and (D) subsequent to chemotherapy. (E) Representative image of case 3 demonstrating no response prior to chemotherapy and (F) subsequent to chemotherapy. (G) Representative image of case 4 demonstrating no response prior to chemotherapy and $(\mathrm{H})$ subsequent to chemotherapy. 
A

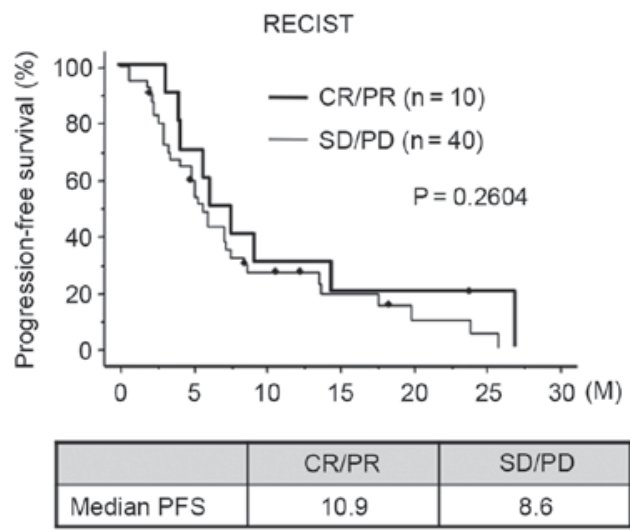

C

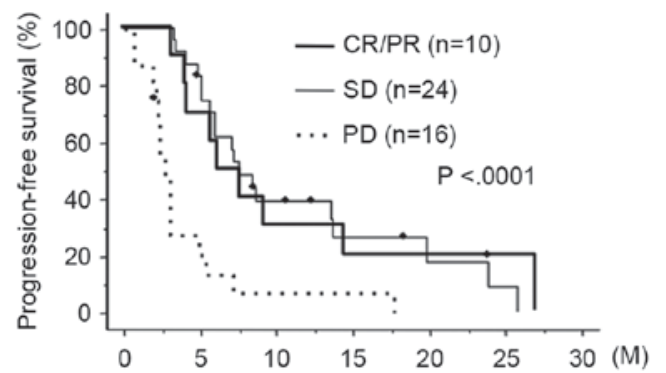

\begin{tabular}{|l|c|c|c|}
\hline & CR/PR & SD & PD \\
\hline Median PFS & 11.5 & 11.0 & 4.0 \\
\hline
\end{tabular}

B

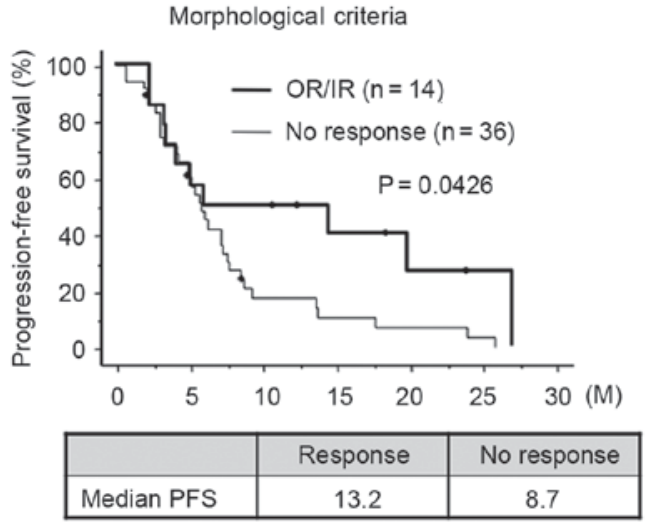

D

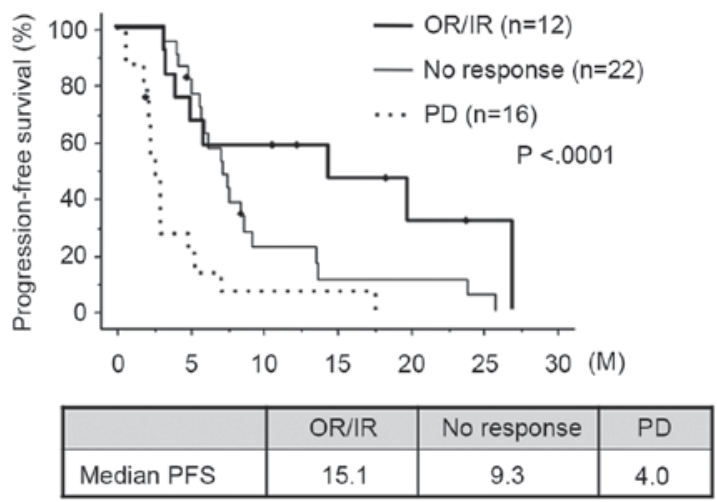

Figure 2. PFS estimated by RECIST and morphological criteria. (A) Comparison of PFS between patients showing CR/PR and SD/PD estimated by RECIST. (B) Comparison of PFS between patients showing OR/IR and NR estimated by morphological criteria. (C) Comparison of PFS between CR/PR, SD and PD. Morphological criteria enhanced the difference in PFS between patients with OR/IR and those with NR. (D) Comparison of PFS between OR/IR, no response and PD. Bevacizumab may exhibit antitumor effects without reducing tumor size and morphological criteria may detect a cytostatic effect of bevacizumab. PFS, progression-free survival; CR, complete response; PR, partial response; SD, stable disease; PD, progressive disease; RECIST, Response Evaluation Criteria in Solid Tumors; NR, no response; IR, incomplete response; OR, optimal response.

the other groups using RECIST and morphological criteria. The RECIST criteria did not show any significant difference in PFS time between patients with CR/PR and SD (11.5 months for CR/PR vs. 11.0 months for SD; Fig. 2C). Morphological criteria, however, revealed an increase in the difference in PFS time between those patients with OR/IR and those with NR (15.1 months for OR/IR vs. 9.3 months for NR; $\mathrm{P}<0.0001$; Fig. 2D). Among those patients who had SD, those treated with bevacizumab had improved median PFS times compared with those who did not receive bevacizumab (13.1 months for bevacizumab and chemotherapy vs. 10.0 months for chemotherapy alone; $\mathrm{P}=0.3415)$. This indicated that bevacizumab may exhibit an antitumor effect that does not result in a reduction in tumor size or morphological criteria. Comparison of the survival curves between the OR/IR and NR groups showed that they remained close to each other up to 6 months after chemotherapy (the second period of CT evaluation), but the curves then diverged from each other subsequent to this date. The median PFS was $>15$ months for patients classified as having an OR or IR. These results indicated that tumors in patients showing OR/IR AT 6 months' post-treatment were oncologically stable, which would make the patients candidates for surgical intervention, including rescue liver resection (Fig. 3).

Univariate analysis. Univariate analysis revealed that morphological response was the only significant prognostic factor for

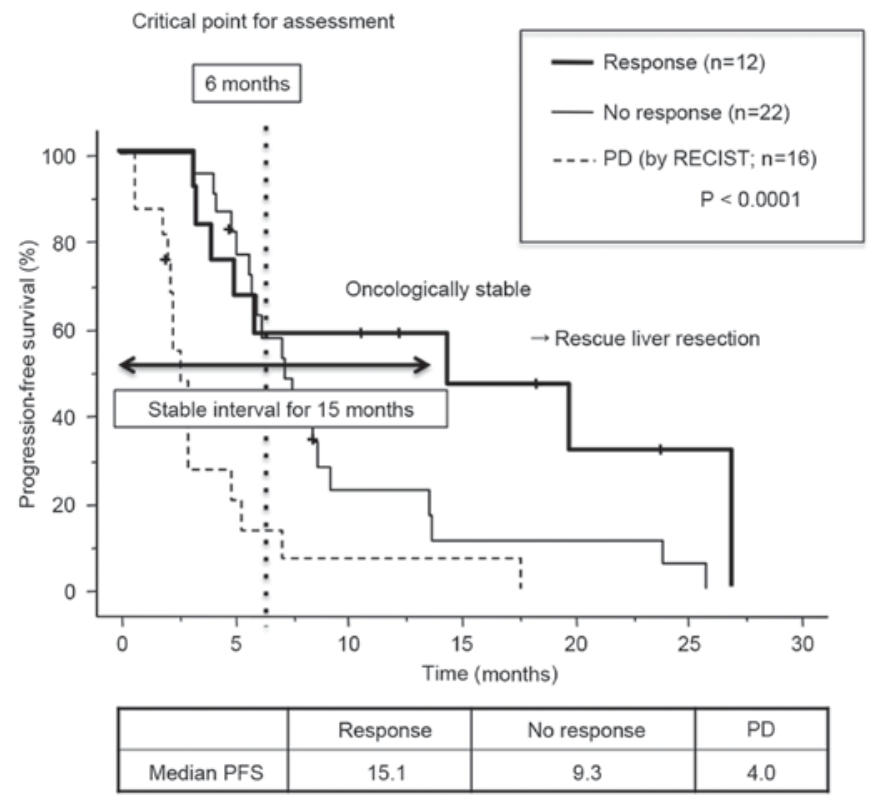

Figure 3. Comparison of PFS between the OR/IR, NR and PD groups. The survival curves of the OR/IR and NR groups were close to each other at 6 months after initial treatment, followed by a large difference. Median PFS $>15$ months was observed in the patients with OR/IR. These results indicated that tumors in the patients showing OR/IR for at least 6 months were oncologically stable and they may be candidates for surgical intervention, including rescue liver resection. PFS, progression-free survival; OR, optimal response; IR, incomplete response; NR, no response; $\mathrm{PD}$, progressive disease. 
Table II. Univariate analysis concerning the prediction of progression free survival in the 50 patients.

\begin{tabular}{|c|c|c|c|}
\hline Factors & $\mathrm{n}$ & Coefficient $(95 \%$ CI) & P-value \\
\hline Gender & & $0.816(0.419-1.588)$ & 0.549 \\
\hline Male & 29 & & \\
\hline Female & 21 & & \\
\hline Primary tumor site & & $0.758(0.412-1.393)$ & 0.372 \\
\hline Colon & 28 & & \\
\hline Rectum & 22 & & \\
\hline Occurrence of metastasis & & $1.436(0.769-2.685)$ & 0.256 \\
\hline Simultaneous & 34 & & \\
\hline Metachronous & 16 & & \\
\hline Number of liver metastasis & & $1.159(0.630-2.133)$ & 0.634 \\
\hline$<5$ & 27 & & \\
\hline$\geq 5$ & 23 & & \\
\hline Extra hepatic lesions & & $0.600(0.321-1.123)$ & 0.110 \\
\hline Positive & 30 & & \\
\hline Negative & 20 & & \\
\hline Size of largest metastasis, $\mathrm{cm}$ & & $0.886(0.479-1.639)$ & 0.701 \\
\hline$<5$ & 29 & & \\
\hline$\geq 5$ & 21 & & \\
\hline Bevacizumab & & $0.889(0.462-1.710)$ & 0.725 \\
\hline Yes & 19 & & \\
\hline No & 31 & & \\
\hline RECIST & & $0.565(0.266-1.198)$ & 0.136 \\
\hline $\mathrm{CR} / \mathrm{PR}$ & 10 & & \\
\hline $\mathrm{SD} / \mathrm{PD}$ & 40 & & \\
\hline Morphological change & & $2.131(1.005-4.517)$ & $0.048^{\mathrm{a}}$ \\
\hline Response & 14 & & \\
\hline No response & 36 & & \\
\hline
\end{tabular}

${ }^{\mathrm{a}} \mathrm{P}<0.05$. CI, confidence interval; $\mathrm{CR}$, complete response; $\mathrm{PR}$, partial response; $\mathrm{SD}$, stable response; PD, progressive disease.

PFS following chemotherapy (Table II), therefore multivariate analysis was not carried out.

Representative optimal response case. A 60-year-old man presented with CRC and unresectable liver metastasis. The CT images shown in Fig. 4A and B indicate that the patient had multiple liver metastases. The tumors appeared to exhibit heterogeneous attenuation with a thick, poorly-defined tumor-liver interface, which placed the patient in group 3, based on the morphological criteria. The patient was subsequently treated with mFOLFOX6 and bevacizumab, including $200 \mathrm{mg} / \mathrm{m}^{2}$ folinic acid, $400 \mathrm{mg} / \mathrm{m}^{2} 5-\mathrm{FU}$ and $85 \mathrm{mg} / \mathrm{m}^{2}$ oxaliplatin on day 1 and $5 \mathrm{mg} / \mathrm{kg}$ bevacizumab on day 1 and q14d, followed by $46 \mathrm{~h}$ of continuous infusion with $2,400 \mathrm{mg} / \mathrm{m}^{2}$ 5-FU on days 1 and 2, following resection of the ascending colon. After 2 months, the tumors in the liver had changed to become classified as homogeneous low attenuation masses with a thin, sharply-defined tumor-liver interface, which now placed the patient in group 1 (Fig. 4C and D). At the same time, the liver tumors demonstrated an OR; however, during the study, tumors that were OR were not classified as being oncologically stable and resectable, and as such, the patient continued receiving chemotherapy. At 16 months post-chemotherapy, the size of tumors were reduced (Fig. 4E and F) and showed no accumulation on positron emission tomography-CT (data not shown), therefore, a resection of the liver tumors was performed. Fig. 5A-C show the pathological features of the tumor, which consisted of necrosis, granulation and fibrosis, with a clear borderline between the tumor tissue and the non-tumor tissue. Fig. 5D shows the radiological imaging of the tumor with morphological response, which may have been a result of pathological change induced by bevacizumab. While the tumor exhibited a major response according to the tumor regression grade (19), it was revealed to possess living tumor cells at the edge of the necrotic tissue. The living tumor cells were distributed in a way that was consistent with the area of vascular reconstruction of a mouse xenograft model induced by the anti-vascular endothelial growth factor (VEGF) antibody (Fig. 5E and F) in a previous study (20). Therefore, the pathological change may have been a result of the antitumor effect of the bevacizumab that the patient received. 
A

C

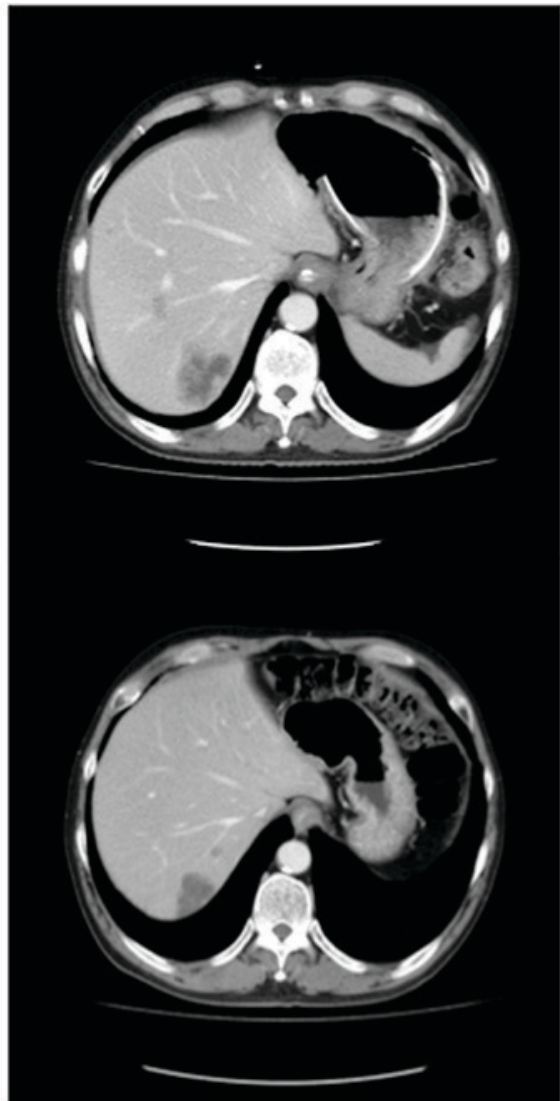

$\mathrm{E}$

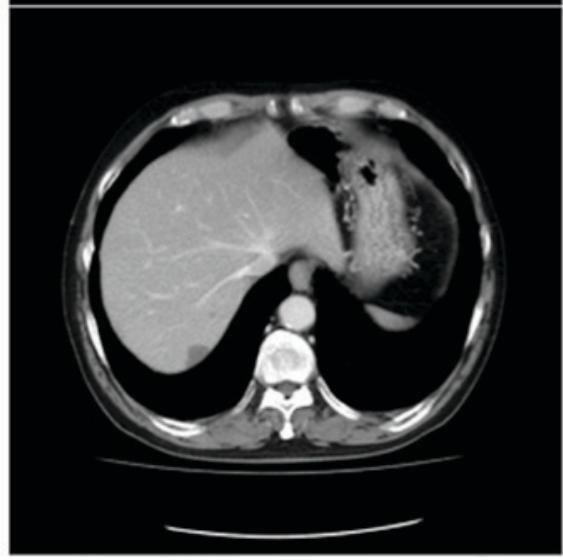

B

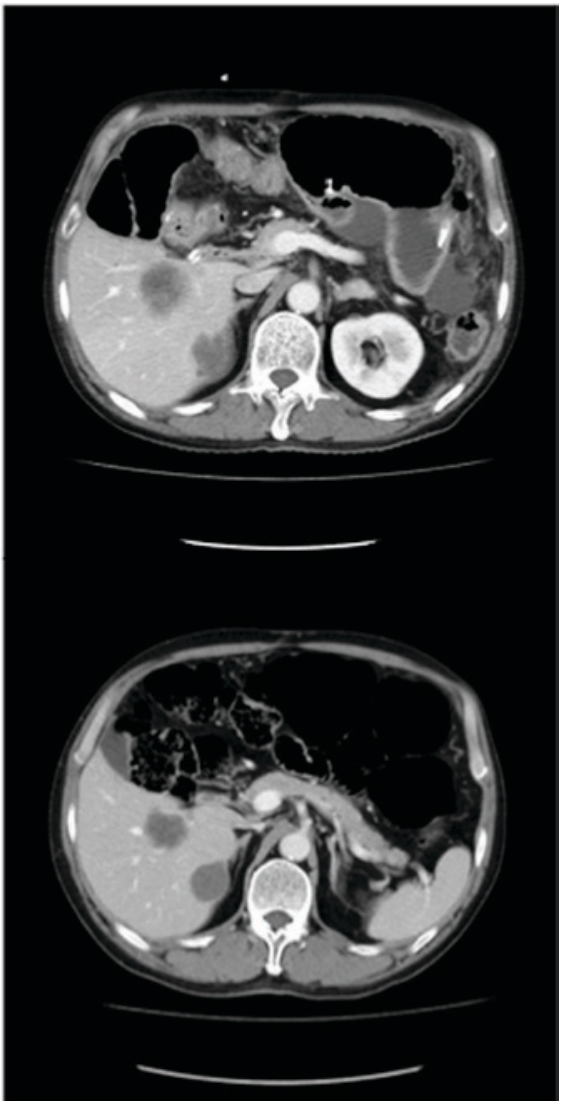

$\mathrm{F}$

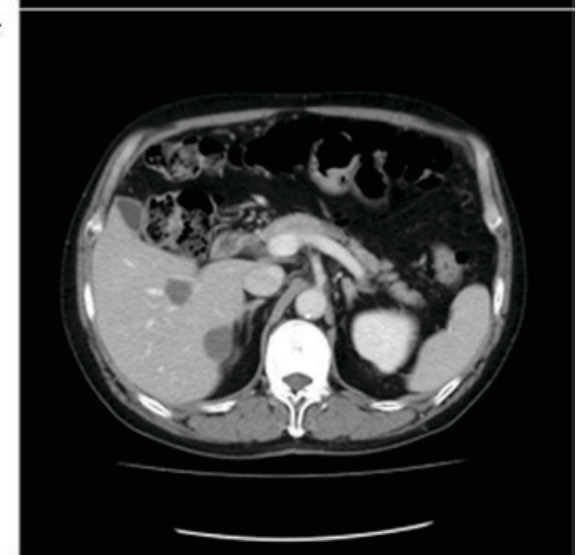

Figure 4. Changes in morphology in computed tomography imaging prior to and following chemotherapy. (A and B) Changes in morphology prior to chemotherapy. Changes in morphology (C and D) 2 months after chemotherapy and (E and F) 16 months after chemotherapy.

\section{Discussion}

The present study demonstrated that patients with mCRC with liver metastasis showing OR/IR within 6 months of chemotherapy were oncologically stable, which made the patients candidates for rescue liver resection. Assessment of the morphological response contributed to the selection of the therapeutic strategy, which included surgical intervention for patients with mCRC who underwent chemotherapy for their initially unresectable tumors.

Assessment of morphological response has been reported to be a good predictor of therapeutic outcomes for patients undergoing chemotherapy $(16-18,21,22)$, whereas conventional size-based criteria, such as RECIST, may be limited in assessing the response of the patient to biological agents that exhibit a cytostatic mechanism of action. The present data revealed that the prognostic advantage of an optimal morphological response of patients with mCRC undergoing chemotherapy with bevacizumab was consistent with results of previous studies (16-18). Furthermore, the present study provides evidence for the clinical application of morphological criteria in selecting the therapeutic strategy for those patients who most likely benefit from surgery.

Advances in combination chemotherapy with biological agents have improved the response rates of patients and may reduce the size of tumors (4). This allows liver resection of patients with mCRC with borderline resectable, as well as unresectable, liver metastasis. In the present study, chemotherapy was used to treat the primary tumor prior to its resection, which was then followed by a hepatectomy.

The limitation of this strategy includes the lack of a clear definition of what constitutes a resectable liver tumor (3). Technical considerations pertaining to the resectability of the tumors may 

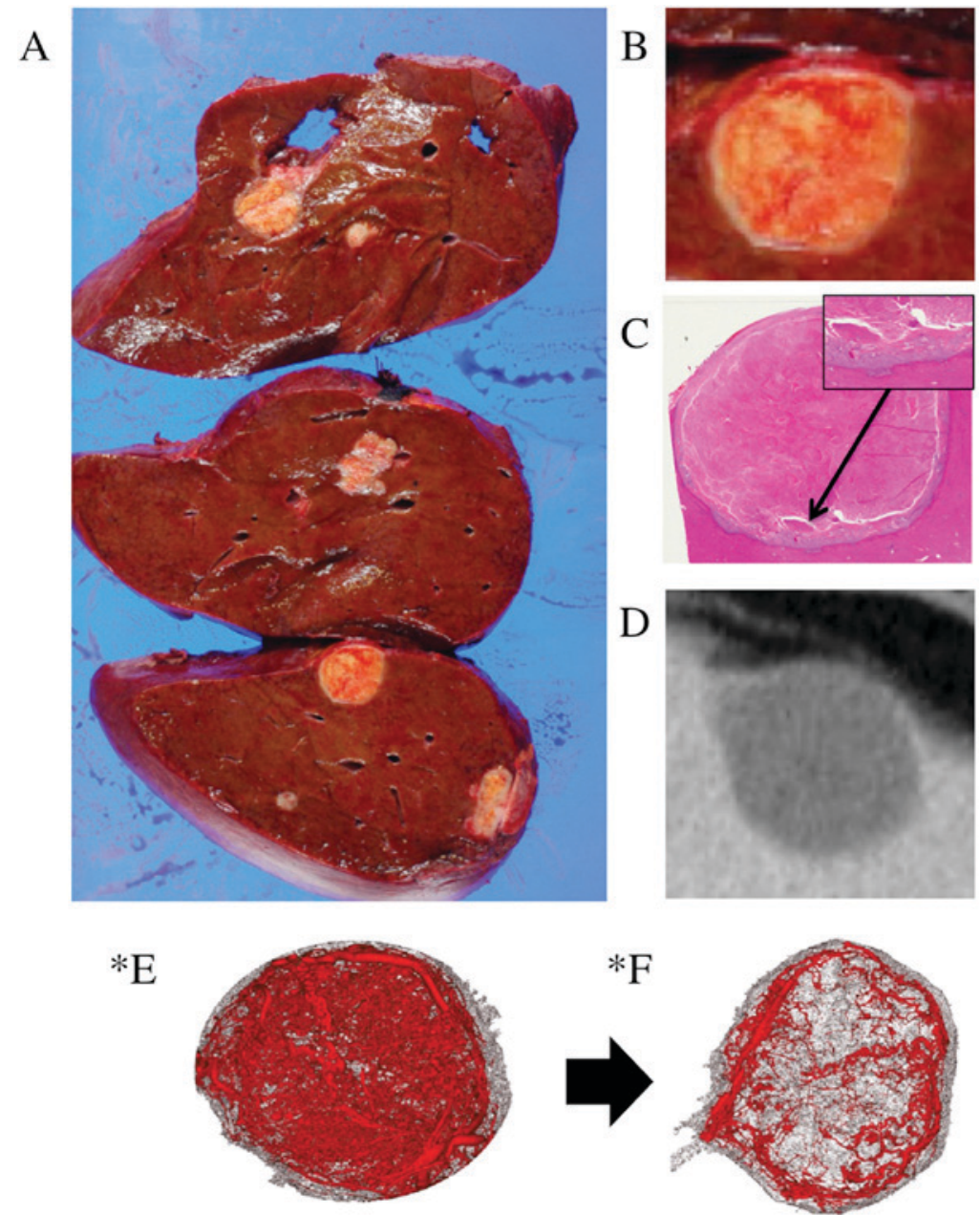

Figure 5. Comparison of features of resected specimens of the tumor, computed tomography imaging and vascular reconstruction of the xenograft in mouse model induced by anti-VEGF antibody. (A) Macroscopic results of the resected liver specimens. (B) Magnified macroscopic results (magnification, x3) of the tumor with clearly defined borders. (C) Pathological findings of the tumor with fibrous and necrotic changes. The tumor harbors living cells at the edge of the necrotic tissues (black arrow). (D) Radiological imaging of the tumor (E) Vascular reconstruction of the xenograft in mouse model induced by anti-VEGF antibody prior to and (F) following treatment. "Refers to (12). The radiological imaging of the tumor with morphological response may be a result of pathological change by bevacizumab, which lead to vascular reconstruction shown in mouse model.VEGF, vascular endothelial growth factor.

be overcome when there is a reduction in the size of the tumor, although oncological concerns may remain. Several guidelines produced by the European Society for Medical Oncology (23) and the National Comprehensive Cancer Network (24) outline the management of patients with $\mathrm{mCRC}$ and liver metastasis. While these guidelines take into account the number, location and distribution of the liver tumors, there is no consensus on how to apply these characteristics to determine whether surgery is indicated. Patient candidates for liver resection are those unlikely to relapse within a short time period following surgery; that is, the patients must possess oncologically stable tumors. The present study was conducted to examine whether morphological criteria are useful in the selection of patients with oncologically stable tumors. The present data shows that the patients who had an OR/IR presented with stable disease and a median PFS period of 15.1 months. As such, they may be classified as potential candidates for rescue liver resection.

While the decision for resection is clear for patients with an OR/IR, those patients whose tumors had a complete response following chemotherapy should also be considered. Benoist et al (25) reported that persistent macroscopic or microscopic residual disease, or early recurrence in situ, was observed in 55 (83\%) of 66 liver metastases that were classified as having a CR on imaging. Therefore, resectable tumors should be treated with surgical intervention while they can be identified on imaging. The majority of patients who showed a morphological response in the present study were classified at the first or second assessment using CT 3-6 months after chemotherapy. The 6-month point is important, as it is the time when a decision is made on whether surgical intervention for rescue liver resection is appropriate for a patient.

The morphological criteria was first reported in 2009 as a novel surrogate marker for the prognosis of patients with mCRC undergoing chemotherapy, including bevacizumab (16), and was later validated surgically (18) and medically (26) in treated populations. The reasons behind why the morphological response has a predictive value for prognosis in patients treated with bevacizumab should also be considered. It has been reported that the pathological response induced by bevacizumab is associated with patient OS (19). A change in morphology, as determined by CT imaging, includes vascular reconstruction, which is believed to be a response to treatment with bevacizumab. By comparison with the pathological and morphological features of the tumor of the representative 
optimal response case in the present study, living tumor cells were revealed to be distributed within a vascular reconstruction area that was induced by an anti-VEGF antibody in a previous study (20). From the present study, it was revealed that morphological response correctly predicted the pathological change produced by the antitumor effect of bevacizumab, which meant that it had predictive value in the prognosis of the patients treated with bevacizumab.

Although the definition of resectable liver metastases has changed, it has been estimated that $20-30 \%$ of patients with liver metastases are potential candidates for liver resection $(27,28)$. Recent phase III trials have shown that the increased use of liver resection as a treatment option has significantly impacted the survival of the CRC population $(1,2)$. The liver resection rate in more recent phase III trials has been between 10 and $14 \%$ for patients who underwent liver resection $(1,29)$. This means that $10-16 \%$ of patients with mCRC remained excluded from surgical treatment. For these patients, the loss of opportunity to be considered for liver resection means they may become candidates for rescue liver resection by assessment of their morphological response.

In conclusion, the present findings provided evidence for physicians to consider previously un-resectable mCRC patients as candidates for surgical treatment. However, it is important to interpret the present results within the context of the study limitations, such as retrospective analysis and selected population, and additional studies may be undertaken prior to definitive guidelines for their clinical application being made.

\section{Acknowledgements}

The present study was supported in part by a grant-in-aid awarded to the post graduate students from Jichi Medical University, a grant-in-aid from the Ministry of Education, Culture, Sports, Science and Technology (grant no. JP16K10514), and the JKA Foundation through its promotion funds from the Keirin Race (grant no. 27-1-068).

\section{References}

1. Venook AP, Niedzwiecki D, Lenz HJ, Innocenti F, Mahoney MR, O'Neil BH, Shaw JE, Polite BN, Hochster HS, Atkins JN, et al: CALGB/SWOG 80405: Phase III trial of irinotecan/5-FU/leucovorin (FOLFIRI) or oxaliplatin/5-FU/leucovorin (mFOLFOX6) with bevacizumab (BV) or cetuximab (CET) for patients (pts) with KRAS wild-type (wt) untreated metastatic adenocarcinoma of the colon or rectum (MCRC). J Clin Oncol 32 (Suppl): 5, 2014.

2. Heinemann V, von Weikersthal LF, Decker T, Kiani A, Vehling-Kaiser U, Al-Batran SE, Heintges T, Lerchenmüller C, Kahl C, Seipelt G, et al: FOLFIRI plus cetuximab versus FOLFIRI plus bevacizumab as first-line treatment for patients with metastatic colorectal cancer (FIRE-3): A randomised, open-label, phase 3 trial. Lancet Oncol 15: 1065-1075, 2014.

3. Siriwardena AK, Mason JM, Mullamitha S, Hancock HC and Jegatheeswaran S: Management of colorectal cancer presenting with synchronous liver metastases. Nat Rev Clin Oncol 11: 446-459, 2014.

4. Kopetz S, Chang GJ, Overman MJ, Eng C, Sargent DJ, Larson DW, Grothey A, Vauthey JN, Nagorney DM and McWilliams RR: Improved survival in metastatic colorectal cancer is associated with adoption of hepatic resection and improved chemotherapy. J Clin Oncol 27: 3677-3683, 2009.

5. Fong Y, Fortner J, Sun RL, Brennan MF and Blumgart LH: Clinical score for predicting recurrence after hepatic resection for metastatic colorectal cancer: Analysis of 1001 consecutive cases. Ann Surg 230: 309321, 1999.
6. Gayowski TJ, Iwatsuki S, Madariaga JR, Selby R, Todo S, Irish W and Starzl TE: Experience in hepatic resection for metastatic colorectal cancer: Analysis of clinical and pathologic risk factors. Surgery 116: 703-711, 1994.

7. Kato T, Yasui K, Hirai T, Kanemitsu Y, Mori T, Sugihara K, Mochizuki $\mathrm{H}$ and Yamamoto J: Therapeutic results for hepatic metastasis of colorectal cancer with special reference to effectiveness of hepatectomy: Analysis of prognostic factors for 763 cases recorded at 18 institutions. Dis Colon Rectum 46 (10 Suppl): 22-31, 2003.

8. Nordlinger B, Guiguet M, Vaillant JC, Balladur P, Boudjema K, Bachellier P and Jaeck D: Surgical resection of colorectal carcinoma metastases to the liver. A prognostic scoring system to improve case selection, based on 1568 patients. Association Française de Chirurgie. Cancer 77: 1254-1262, 1996.

9. Viganò L, Capussotti L, Majno P, Toso C, Ferrero A, De Rosa G, Rubbia-Brandt L and Mentha G: Liver resection in patients with eight or more colorectal liver metastases. Br J Surg 102: 92-101, 2015.

10. Wei AC, Greig PD, Grant D, Taylor B, Langer B and Gallinger S: Survival after hepatic resection for colorectal metastases: A 10-year experience. Ann Surg Oncol 13: 668-676, 2006.

11. Yi JH, Kim H, Jung M, Shin SJ, Choi JS, Choi GH, Baik SH, Min BS, Kim NK and Ahn JB: Prognostic factors for disease-free survival after preoperative chemotherapy followed by curative resection in patients with colorectal cancer harboring hepatic metastasis: A single-institute, retrospective analysis in Asia. Oncology 85: 283-289, 2013.

12. Antoch G, Kanja J, Bauer S, Kuehl H, Renzing-Koehler K, Schuette J, Bockisch A, Debatin JF and Freudenberg LS: Comparison of PET, CT, and dual-modality PET/CT imaging for monitoring of imatinib (STI571) therapy in patients with gastrointestinal stromal tumors. J Nucl Med 45: 357-365, 2004.

13. Stroobants S, Goeminne J, Seegers M, Dimitrijevic S, Dupont P, Nuyts J, Martens M, van den Borne B, Cole P, Sciot R, et al: 18FDG-Positron emission tomography for the early prediction of response in advanced soft tissue sarcoma treated with imatinib mesylate (Glivec). Eur J Cancer 39: 2012-2020, 2003.

14. Blanke CD, Demetri GD, von Mehren M, Heinrich MC, Eisenberg B, Fletcher JA, Corless CL, Fletcher CD, Roberts PJ, Heinz D, et al: Long-term results from a randomized phase II trial of standard-versus higher-dose imatinib mesylate for patients with unresectable or metastatic gastrointestinal stromal tumors expressing KIT. J Clin Oncol 26: 620-625, 2008.

15. Therasse P, Arbuck SG, Eisenhauer EA, Wanders J, Kaplan RS, Rubinstein L, Verweij J, Van Glabbeke M, van Oosterom AT, Christian MC, et al: New guidelines to evaluate the response to treatment in solid tumors. european organization for research and treatment of cancer, national cancer institute of the United States, national cancer institute of Canada. J Natl Cancer Inst 92: 205-216, 2000.

16. Chun YS, Vauthey JN, Boonsirikamchai P, Maru DM, Kopetz S, Palavecino M, Curley SA, Abdalla EK, Kaur H, Charnsangavej $\mathrm{C}$ and Loyer EM: Association of computed tomography morphologic criteria with pathologic response and survival in patients treated with bevacizumab for colorectal liver metastases. Jama 302: 2338-2344, 2009.

17. Nishioka Y, Shindoh J, Yoshioka R, Gonoi W, Abe H, Okura N, Yoshida S, Oba M, Hashimoto M, Watanabe G, et al: Radiological morphology of colorectal liver metastases after preoperative chemotherapy predicts tumor viability and postoperative outcomes. J Gastrointest Surg 19: 1653-1661, 2015.

18. Shindoh J, Loyer EM, Kopetz S, Boonsirikamchai P, Maru DM, Chun YS, Zimmitti G, Curley SA, Charnsangavej C, Aloia TA and Vauthey JN: Optimal morphologic response to preoperative chemotherapy: An alternate outcome end point before resection of hepatic colorectal metastases. J Clin Oncol 30: 4566-4572, 2012.

19. Klinger M, Tamandl D, Eipeldauer S, Hacker S, Herberger B, Kaczirek K, Dorfmeister M, Gruenberger B and Gruenberger T: Bevacizumab improves pathological response of colorectal cancer liver metastases treated with XELOX/FOLFOX. Ann Surg Oncol 17: 2059-2065, 2010.

20. O'Connor JP, Carano RA, Clamp AR, Ross J, Ho CC, Jackson A, Parker GJ, Rose CJ, Peale FV, Friesenhahn M, et al: Quantifying antivascular effects of monoclonal antibodies to vascular endothelial growth factor: Insights from imaging. Clin Cancer Res 15: 6674-6682, 2009. 
21. Choi H, Charnsangavej C, Faria SC, Macapinlac HA, Burgess MA, Patel SR, Chen LL, Podoloff DA and Benjamin RS: Correlation of computed tomography and positron emission tomography in patients with metastatic gastrointestinal stromal tumor treated at a single institution with imatinib mesylate: Proposal of new computed tomography response criteria. J Clin Oncol 25: 1753-1759, 2007.

22. Shindoh J, Chun YS, Loyer EM and Vauthey JN: Non-size-based response criteria to preoperative chemotherapy in patients with colorectal liver metastases: The morphologic response criteria. Curr Colorectal Cancer Rep 9: 198-202, 2013.

23. Schmoll HJ, Van Cutsem E, Stein A, Valentini V, Glimelius B, Haustermans K, Nordlinger B, van de Velde CJ, Balmana J, Regula J, et al: ESMO consensus guidelines for management of patients with colon and rectal cancer. a personalized approach to clinical decision making. Ann Oncol 23: 2479-2516, 2012.

24. NCCN Clinical Practice Guidelines in Oncology: Colon Cancer. http://www.nccn.org/professionals/physician_gls/f_guidelines.asp. Accessed January 1, 2016.

25. Benoist S, Brouquet A, Penna C, Julié C, El Hajjam M, Chagnon S, Mitry E, Rougier P and Nordlinger B: Complete response of colorectal liver metastases after chemotherapy: Does it mean cure? J Clin Oncol 24: 3939-3945, 2006
26. Yoshita H, Hosokawa A, Ueda A, Ando T, Kajiura S, Kato H, Kawabe H, Tomizawa G, Horikawa N, Yabuhita K, et al: Predictive value of optimal morphologic response to first-line chemotherapy in patients with colorectal liver metastases. Digestion 89: 43-48, 2014

27. Garden OJ, Rees M, Poston GJ, Mirza D, Saunders M, Ledermann J, Primrose JN and Parks RW: Guidelines for resection of colorectal cancer liver metastases. Gut 55 (Suppl 3): iiil-8, 2006

28. Stangl R, Altendorf-Hofmann A, Charnley RM and Scheele J: Factors influencing the natural history of colorectal liver metastases. Lancet 343: 1405-1410, 1994.

29. Schwartzberg LS, Rivera F, Karthaus M, Fasola G, Canon JL, Hecht JR, Yu H, Oliner KS and Go WY: PEAK: A randomized, multicenter phase II study of panitumumab plus modified fluorouracil, leucovorin and oxaliplatin (mFOLFOX6) or bevacizumab plus mFOLFOX6 in patients with previously untreated, unresectable, wild-type KRAS exon 2 metastatic colorectal cancer. J Clin Oncol 32: 2240-2247, 2014 